Supporting Information for:

\title{
Using Laser-Induced Thermal Voxels to Pattern Diverse Materials at the Solid-Liquid Interface
}

\author{
Lauren D. Zarzar ${ }^{\dagger, *}$, B. S. Swartzentruber ${ }^{\ddagger}$, Brian F. Donovan ${ }^{\S}$, Patrick E. Hopkins ${ }^{\S}$ and \\ Bryan Kaehr ${ }^{\perp, \#, *}$ \\ ${ }^{\dagger}$ Department of Materials Science and Engineering, Department of Chemistry, The Pennsylvania State University, \\ University Park, Pennsylvania 16802, United States; ${ }^{\ddagger}$ Center for Integrated Nanotechnologies, Sandia National \\ Laboratories, Albuquerque, NM 87545, United States. ${ }^{\S}$ Department of Mechanical and Aerospace Engineering, \\ University of Virginia, Charlottesville, Virginia 22904, United States; ${ }^{\perp}$ Advanced Materials Laboratory, Sandia \\ National Laboratories, Albuquerque, New Mexico 87185, United States; "Department of Chemical and Biological \\ Engineering, the University of New Mexico, Albuquerque, New Mexico 87131, United States
}

*correspondence to: 1dz4@psu.edu; bjkaehr@sandia.gov 


\section{$\underline{\text { Experimental Procedures }}$}

Precursor and working concentrations. Ammonium tetrachloroplatinate (II), 0.5-0.7 M; ammonium tetrachloropalladate (II), 0.5-0.7 M; ammonium hexachlororhodate (III), saturated solution; ammonium hexachloroiridate (III) monohydrate, saturated solution; cupric sulfate pentahydrate, $1 \mathrm{M}$; potassium permanganate, $0.7 \mathrm{M}$; cobalt (II) chloride hexahydrate, $0.7 \mathrm{M}$; chromium (III) chloride hexahydrate, $1 \mathrm{M}$; nickel (II) nitrate hexahydrate, $1 \mathrm{M}$; ammonium molybdate tetrahydrate, saturated solution; vanadium (IV) oxide sulfate, $1 \mathrm{M}$; ammonium hexachlororuthenate (IV), $0.25 \mathrm{M}$; ammonium iron (III) oxalate trihydrate, $1 \mathrm{M}$; iron (II) chloride tetrahydrate, saturated solution; potassium ferrate (IV), $0.5 \mathrm{M}$; iron (II) sulfate heptahydrate, $0.5 \mathrm{M}$. The above precursors were all dissolved in deonized $\mathrm{H}_{2} \mathrm{O}(18.2 \mathrm{M} \Omega \bullet \mathrm{cm}$ at $25^{\circ} \mathrm{C}$ ) to yield working concentrations. For fabrication of Ni metal structures, $1 \mathrm{M}$ nickel (II) nitrate hexahydrate was dissolved in ethylene glycol. The laser power used for these experiments was between $\sim 5-50 \mathrm{~mW}$ as measured at the sample. The volumes of precursor used for fabrication resulted in liquid layer depths of $>2 \mathrm{~mm}$ ( $\sim 3$ orders of magnitude greater the height of the thermal reaction zone).

Laser experimental setup. All experiments employed a titanium:sapphire laser (Tsunami; Spectra Physics) operating at $750 \mathrm{~nm}$ in either pulsed (for deposition of platinum pads ${ }^{1}$ ) or continuous wave (CW) mode. The laser output was adjusted by using optics to approximately fill the back aperture of an oil-immersion objective (Nikon 100× Fluor, 1.3 numerical aperture) situated on a Nikon inverted microscope. Images were acquired using an EMCCD camera (iXon, Andor) and the laser light was filtered from the camera optics using a colored glass band-pass filter (Thorlabs, BG39). Laser powers used for these experiments were between $\sim 5-50 \mathrm{~mW}$ as measured at the sample and obtained by attenuating the laser beam using a half-wave plate/polarizing beam-splitter pair. The $1 / e^{2}$ radius of the focused laser spot was $\sim 500 \mathrm{~nm}$ and the exposure area could be enlarged to a desired size by raster-scanning the beam using an X/Y open frame scan head. For the materials and structures shown in Figure 2, the scan rate was 180 $\mathrm{Hz}$ (for the $\mathrm{Y}$ direction) and the area was scanned using a single pass $(0.1-0.2 \mathrm{~Hz}$ for the $\mathrm{X}$ direction). For simple lines and shapes, the beam focus was translated in XY using a motorized stage controller (ASI MS-2000) to move the substrate in relation to the laser focus. For more complex patterns, the laser focus was raster scanned with the $\mathrm{X} / \mathrm{Y}$ scanner across a reflectance mask (digital micromirror device) used to define the features of microstructures. ${ }^{2}$

Thermal Model. The steady state temperature rise $(\Delta \mathrm{T})$ at the metal absorber edge used to build the thermal model described in the paper was calculated using the solution for the low frequency limit of the laser modulation ${ }^{3}$

$$
\Delta T=\frac{\alpha P_{0}}{2 r_{0} \kappa \sqrt{\pi}}
$$


where $P_{0}$ is the average power $(\sim 17 \mathrm{~mW}), \alpha$ is the absorptivity (assumed to be 0.3 for Pt at 750 $\mathrm{nm}, \mathrm{w}_{0}$ is the $1 / \mathrm{e}^{2}$ radius of the focused laser spot $(\sim 500 \mathrm{~nm})$, and $\kappa$ is the thermal conductivity of the glass $\left(1.3 \mathrm{Wm}^{-1} \mathrm{~K}^{-1}\right)$ giving a maximum steady state temperature rise of $\sim 2,000 \mathrm{~K}$.

The spatial temperature profiles in the plane of the metal $(\mathrm{Pt})$, across the metal/liquid interfacial region, and in the liquid resulting from this increased temperature rise induced from the absorbed laser power were calculated in MATLAB via a numerical solution to the 2D Heat Equation (Laplace Equation). The numerical input details, mesh size, boundary conditions (which include the thermal boundary conductance) and pertinent thermophysical properties, along with resulting temperature profiles, are given in the main document. More specifically, we solved

$$
\frac{\partial^{2} T}{\partial x^{2}}+\frac{\partial^{2} T}{\partial y^{2}}=0
$$

using a finite difference method. The boundary conditions at the Pt/liquid interface are given in the main document. Using a square mesh grid, our discretized temperature derivatives become

$$
\left.\frac{\partial^{2} T}{\partial x^{2}}\right|_{m, n}=\frac{T_{m+1, n}-2 T_{m, n}+T_{m-1, n}}{(\Delta x)^{2}}
$$

in $\mathrm{x}$, and

$$
\left.\frac{\partial^{2} T}{\partial y^{2}}\right|_{m, n}=\frac{T_{m, n+1}-2 T_{m, n}+T_{m, n-1}}{(\Delta y)^{2}}
$$

in $\mathrm{y}$, where $\mathrm{m}$ and $\mathrm{n}$ are spatial indices in our numerical domain. 


\section{Supporting Figures}
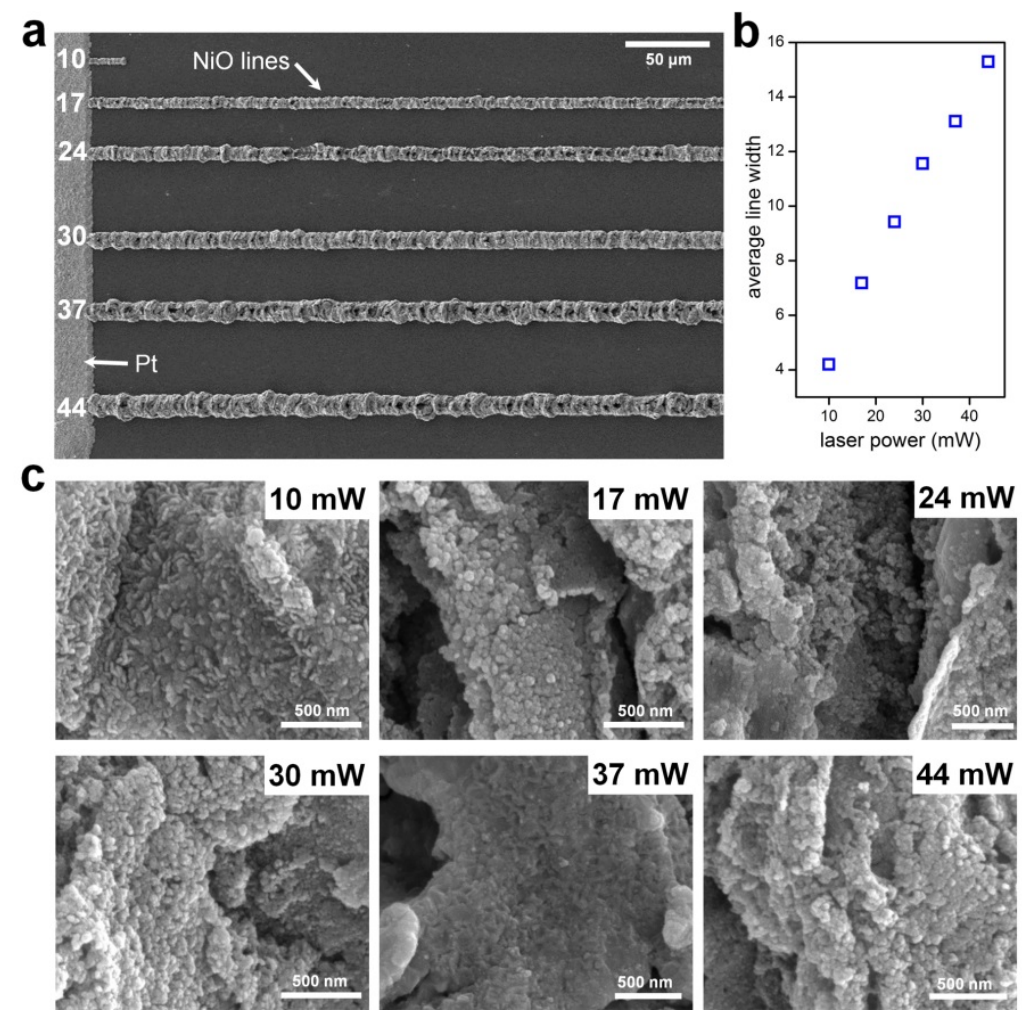

Figure S1. (a) SEM images of $\mathrm{NiO}$ lines drawn with the same scan rate of $10 \mu \mathrm{m} / \mathrm{s}$ and varying laser power (as indicated by the numbers at left, in $\mathrm{mW}$ ). (b) Increasing laser power results in increasing line width of NiO. (c) Top down SEM images of the NiO structures shown in (a).

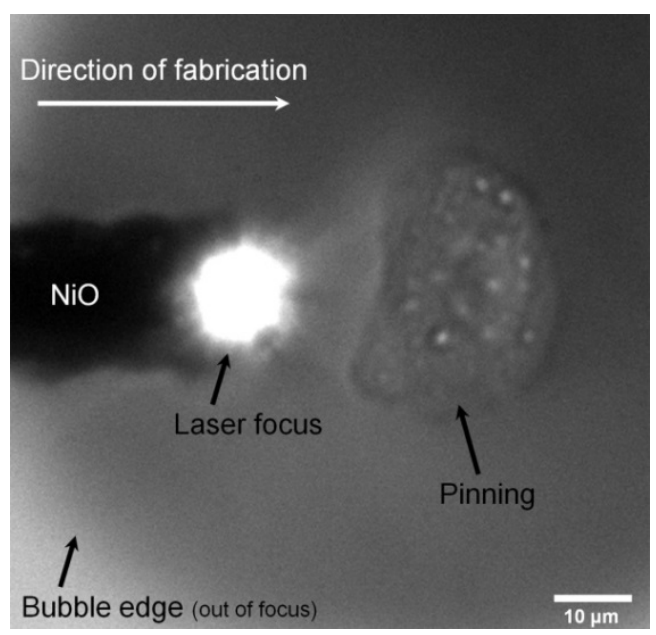

Figure S2. Snapshot of the fabrication process of an NiO structure from a bath solution of $1 \mathrm{M}$ $\mathrm{Ni}\left(\mathrm{NO}_{3}\right)_{2}$ dissolved in water shows broadband light emission (the laser light is filtered out from the camera optics) at the point of fabrication and subsequent boiling of the local solution. Here, the bubble is momentarily pinned to the glass surface in front of the direction of fabrication. 


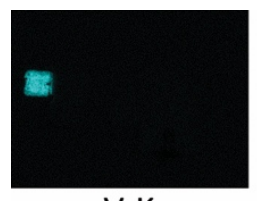

V-K

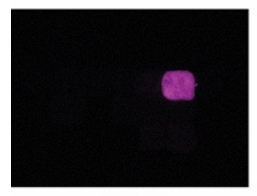

$\mathrm{Ni}-\mathrm{K}$

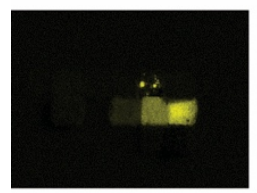

Pd-L

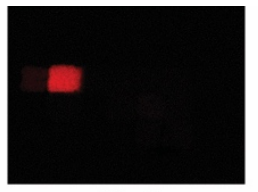

Cr-K

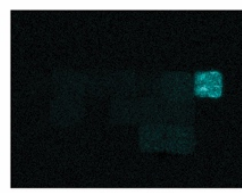

Cu-K

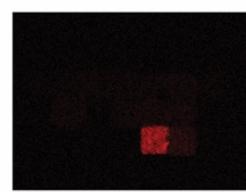

Ir-L

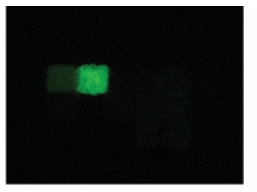

$\mathrm{Mn}-\mathrm{K}$

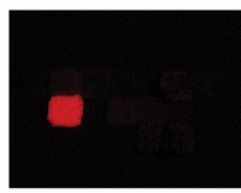

Mo-L

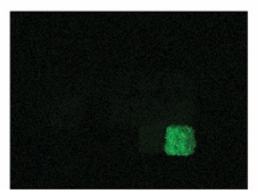

Pt-L

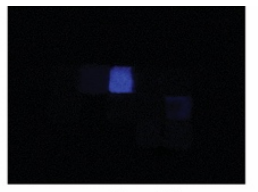

Fe-K

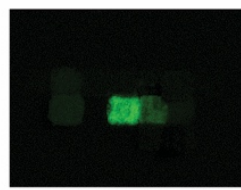

Ru-L

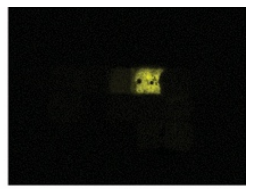

Co-K

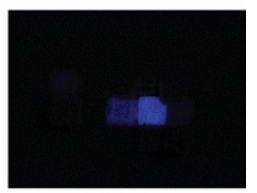

Rh-L

Figure S3. Individual, raw energy dispersive X-ray spectroscopy (EDS) maps of each element and the corresponding emission line ( $\mathrm{K}$ or $\mathrm{L}$ ). These maps were used to create the composite image in Figure 2c. Colors were selected to distinguish between all elements.

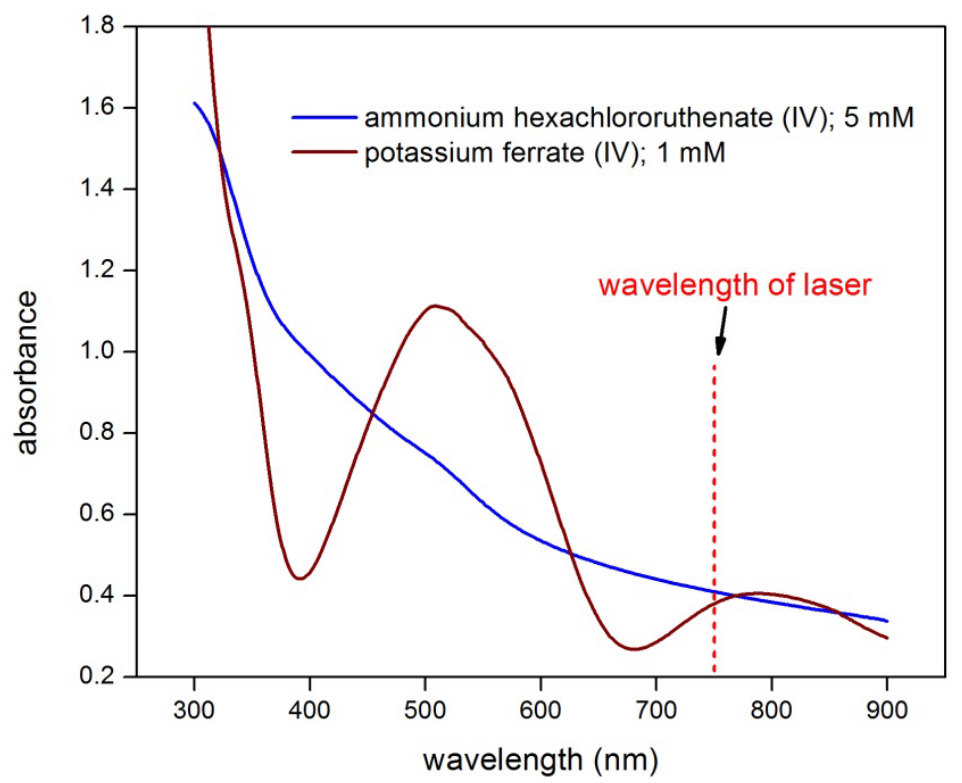

Figure S4. Absorbance spectrum of ruthenium and iron oxide precursors that could be written directly from aqueous solution (without using an absorbing solid edge) via direct absorption of $750 \mathrm{~nm}$ laser light. 


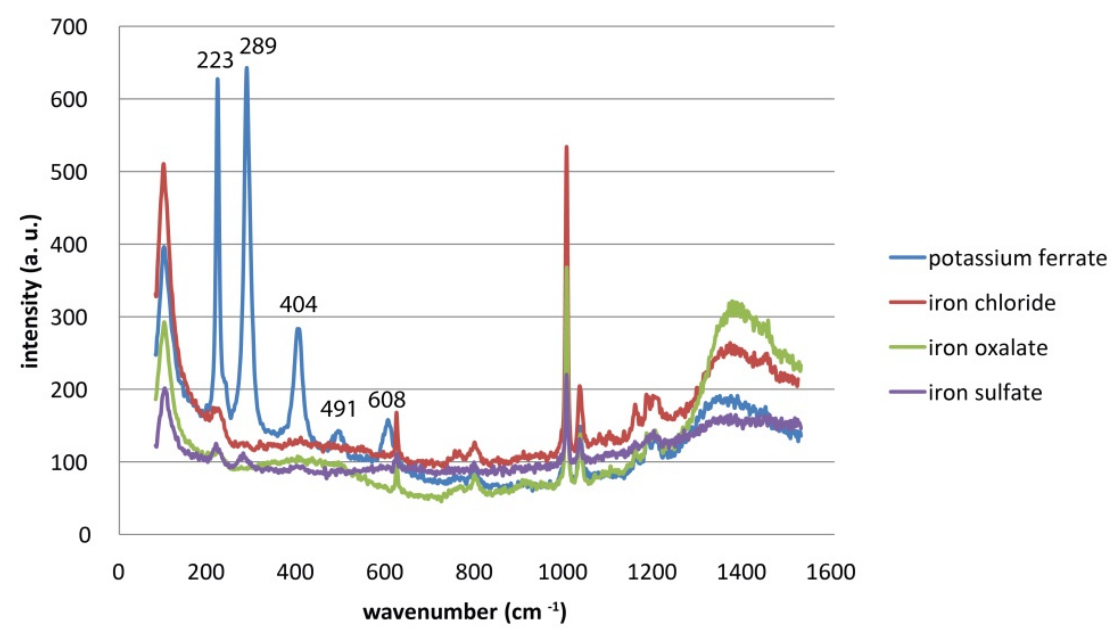

Figure S5. Raman spectra of iron oxide structures from various precursors. Arrows denote peak positions indicative of hematite, $\left(\mathrm{Fe}_{2} \mathrm{O}_{3}\right)$ resulting from fabrication using potassium ferrate. Structures fabricated using the remaining precursors resulted in mixed phase oxides that could not be indexed.

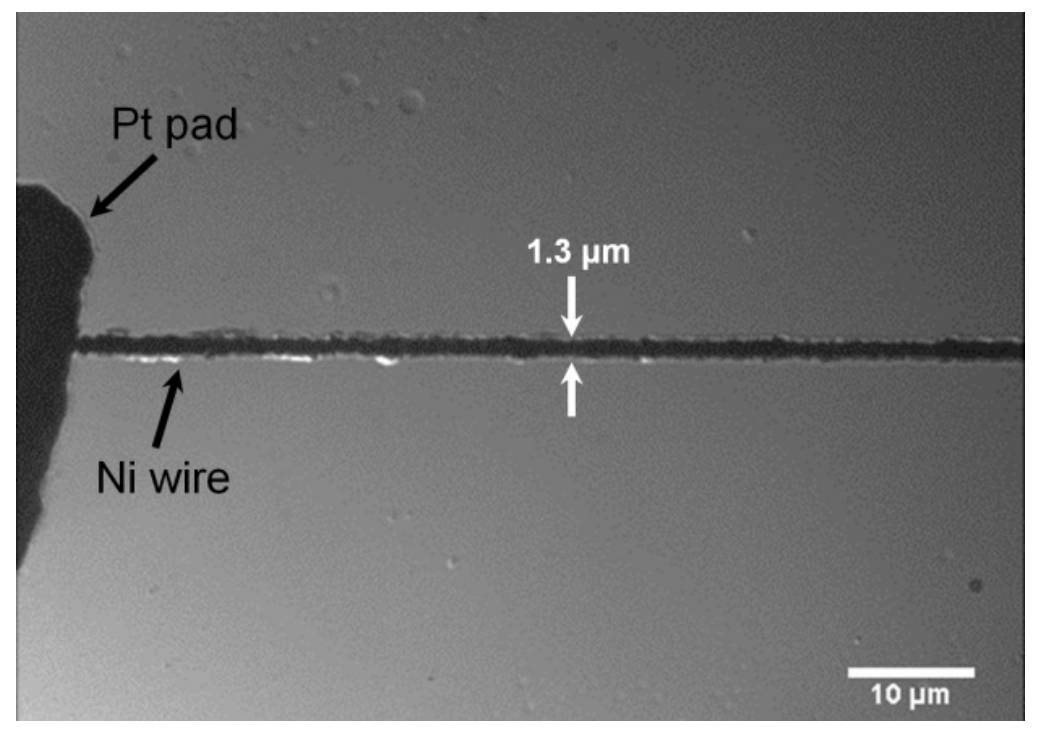

Figure S6. The achieved minimum repeatable line width for Ni metal wire synthesis on a glass substrate at a scan rate of $1 \mathrm{~mm} / \mathrm{s}$. 

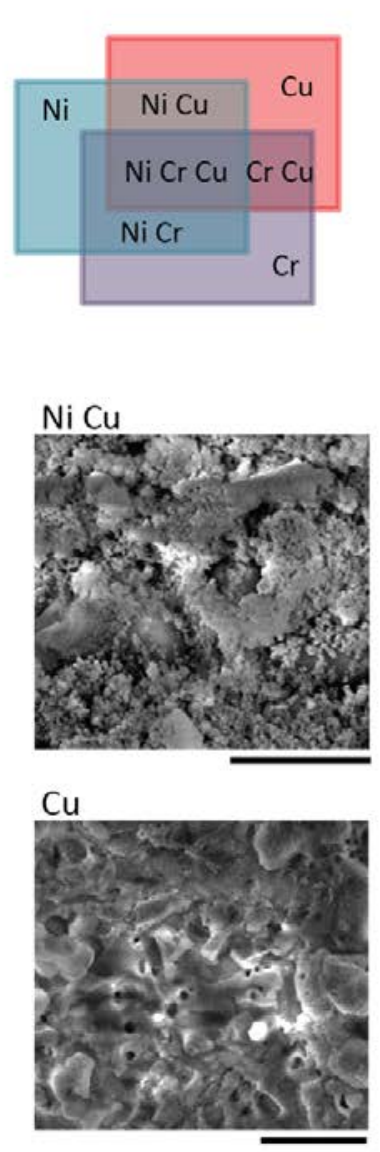
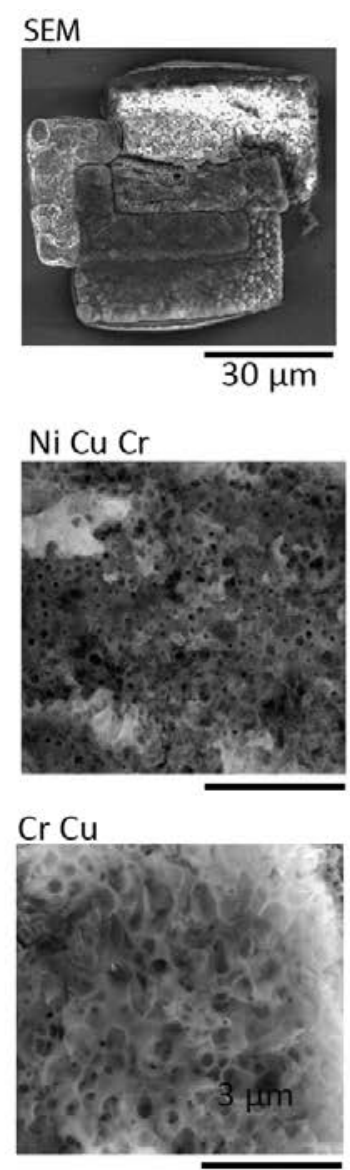
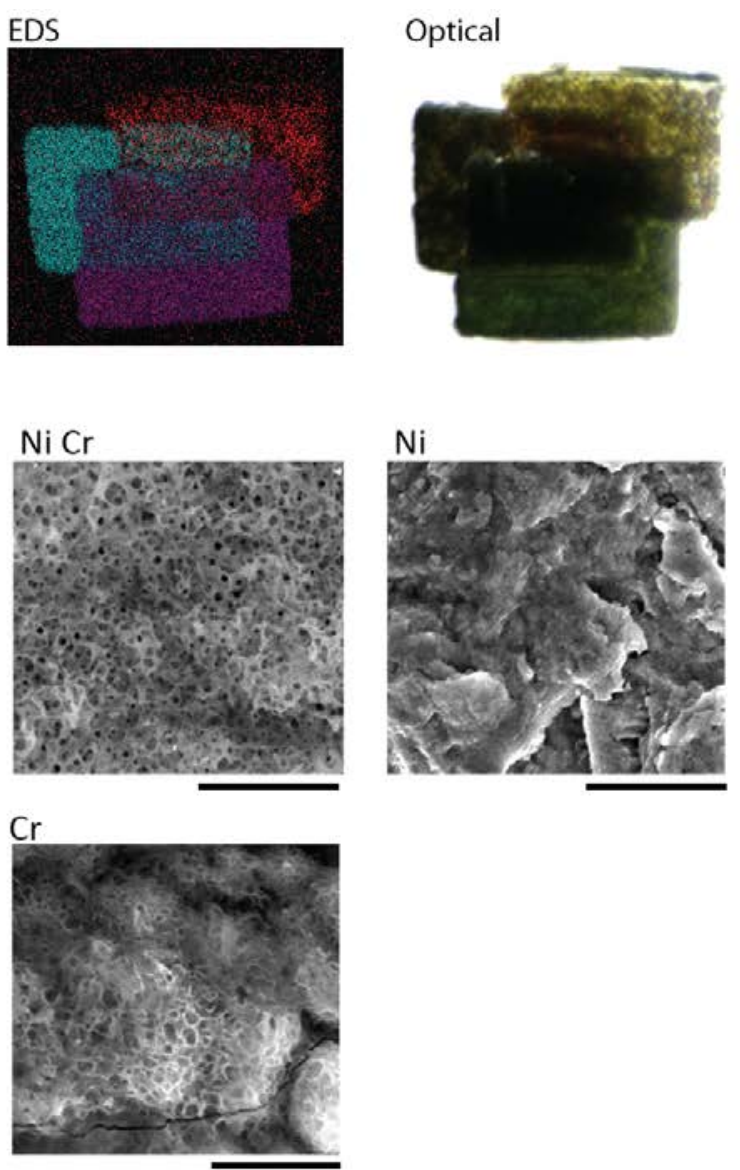

Figure S7. Optical, SEM, and EDS images of a fully iterated and spatially patterned, multielemental system as a concept proof for generation of mixed metal oxide structures using LITV. Each region was fabricated step-wise using mixed metal salt precursors dissolved in water. The EDS spectrum indicates minimal bleed/contamination or mixing of these elements except where desired according to the map (top left panel). Scale $=3 \mu \mathrm{m}$ except where noted otherwise.

\section{References}

1. Zarzar, L. D.; Swartzentruber, B. S.; Harper, J. C.; Dunphy, D. R.; Brinker, C. J.; Aizenberg, J.; Kaehr, B., Multiphoton Lithography of Nanocrystalline Platinum and Palladium for Site-Specific Catalysis in 3D Microenvironments. J. Am. Chem. Soc. 2012, 134, 4007-4010.

2. Nielson, R.; Kaehr, B.; Shear, J. B., Microreplication and Design of Biological Architectures using Dynamic-Mask Multiphoton Lithography. Small 2009, 5 (1), 120 125.

3. Cahill, D. G., Analysis of Heat Flow in Layered Structures for Time-Domain Thermoreflectance. Rev. Sci. Instrum. 2004, 75 (12), 5119-5122. 